\title{
Enfanter, est-ce bien « naturel »? Rite, représentation, fantasme de l'engendrement dans un culte polynésien
}

Françoise Douaire-Marsaudon

\section{(2) OpenEdition \\ Journals}

Édition électronique

URL : http://journals.openedition.org/jso/6022

DOI : $10.4000 /$ jso.6022

ISSN : 1760-7256

Éditeur

Société des océanistes

Édition imprimée

Date de publication : 15 décembre 2010

Pagination : 89-104

ISBN : 978-2-85430-027-7

ISSN : 0300-953x

Référence électronique

Françoise Douaire-Marsaudon, «Enfanter, est-ce bien « naturel » ? Rite, représentation, fantasme de l'engendrement dans un culte polynésien », Journal de la Société des Océanistes [En ligne], 130-131 | 2010, mis en ligne le 15 décembre 2013, consulté le 09 juin 2020. URL : http:// journals.openedition.org/jso/6022 ; DOI : https://doi.org/10.4000/jso.6022 


\title{
Enfanter, est-ce bien « naturel »? Rite, représentation, fantasme de l'engendrement dans un culte polynésien
}

\author{
par
}

Françoise DOUAIRE-MARSAUDON*

\section{RÉSUMÉ}

Dans le corpus mythique des sociétés de Polynésie, il existe un puissant intérêt pour tout ce qui concerne la reproduction et la filiation et, plus particulièrement, pour l'enfantement et la naissance. Cet article propose une contribution à la discussion sur le caractère "naturel » attribué, dans nos sociétés, aux procès associés à l'enfantement, dans la perspective critique ouverte par Bernard Juillerat. L'analyse d'un ensemble mytho-rituel de Polynésie permet de montrer que, dans ces sociétés où la dichotomie naturelculture ne fait pas sens, l'enfantement est conçu comme un événement constitutif de la société, autrement dit « culturel».

MoTS-CLÉS : nature, culture, engendrement, filiation, enfantement, naissance, inceste sacré, ordre sociocosmique

Pour Bernard Juillerat, penser l'imaginaire des sociétés, c'est se donner les moyens d'appréhender «ce curieux mélange de fantasmes individuels et d'idéologie collective» (2001 : 159) qui en fournit en partie le matériau et, pour ce faire, l'anthropologie psychanalytique est non seulement souhaitable mais nécessaire. En plaçant au cœur de ses problématiques la question des rapports entre le sujet (et sa formation) et les faits de culture, elle est, en effet, à même de repérer et d'explorer la part du travail psychique dans l'élaboration des cultures et donc susceptible de comprendre le rapport

\begin{abstract}
Oral traditions in Polynesian societies focus on reproduction and descent, particularly concerning procreation and childbirth. This article discusses the so-called «natural» character which in our own society is attributed to the process of childbirth, and is in line with the critical perspective proposed by Bernard Juillerat. The analysis of a set of Polynesian myths and rites demonstrates that, here, the naturelculture dichotomy does not make sense, and that childbirth represents a specifically cultural event which can be considered to be constitutive for society.
\end{abstract}

KEYWORDS: nature, culture, descent, begetting, childbirth, birth, sacred incest, socio-cosmic order

entre les productions culturelles et la psyché des personnes.

Anthropologue, je n'ai pas fait le choix de l'approche psychanalytique, mais je considère cependant qu'il est difficile de tenir les représentations culturelles et, singulièrement, celles concernant la sexualité, la reproduction, la filiation et la mort, pour « des signes arbitraires, contingents, dont la destinée sociale s'accomplirait indépendamment de leur origine psychique » (Juillerat, $2001: 66$ ). En d'autres termes, à un moment de l'histoire des sciences sociales où l'on cherche à déconstruire l'opposition entre

* Anthropologue, DR2, CREDO-MAP - Université de Provence, Marseille, francoise.douaire@univ-provence.fr 
individu et société - ou à repenser leur relation en termes neufs - et où l'on assiste à un retour en force du sujet, je vois mal comment l'anthropologie, qu'elle soit ou non psychanalytique, pourrait se désintéresser de ce qui lie « la culture aux problématiques du sujet et à ses investissements » (Juillerat, 2004 : 16).

Dans les sociétés de Polynésie où je travaille, il existe un puissant intérêt pour tout ce qui concerne la reproduction et la filiation et, plus précisément, l'engendrement/enfantement et la naissance. Ces préoccupations, telles du moins qu'elles s'expriment dans les corpus mythologiques ou au sein de la vie rituelle, prennent assez régulièrement la forme de récurrences de la symbolique œdipienne, sous des traits propres à ces sociétés, comme on le verra. Mais au-delà de ce simple constat, je voudrais ici, en m'aidant du matériau polynésien, apporter une contribution à la réflexion sur le caractère " naturel » généralement attribué, dans nos sociétés, aux procès entourant l'enfantement - procréation, gestation, accouchement, allaitement - et m'inscrire ainsi dans la perspective critique ouverte par Bernard Juillerat à ce propos (2001 : 110-111).

\section{La « tache blanche » de la relation mère-enfant}

Dans le chapitre IV intitulé "L'atome de parenté est-il soluble dans la psychanalyse » de son livre Penser l'imaginaire. Essais d'anthropologie psychanalytique, Bernard Juillerat revient sur la notion d'atome de parenté de Claude LéviStrauss et sur la " non-qualification des relations entre la mère et l'enfant dans l'atome $»^{1}$. Cette relation mère-enfant avait fait l'objet d'un débat après un exposé d'André Green au séminaire L'identité (Lévi-Strauss, 1983 : 81-107). À André Green, Claude Lévi-Strauss explique que, s'il a fait l'économie de la relation mère-enfant dans l'atome de parenté, c'est parce que :

« les sociétés que nous connaissons normalisent le rapport entre le mari et la femme, $[\ldots]$ entre le frère et la sœur, [...] entre le père et le fils mais ne normalisent pas - en tout cas pas au même degré - la relation entre la mère et ses enfants. » (Lévi-Strauss, 1983 : 100)

Au cours de la discussion, il précise que, s'il ne traite pas de cette relation au sein de l'atome de parenté, c'est «qu'il n'en a pas besoin pour expliquer des conduites collectives» (1983: 101). Comme le lui fit remarquer André Green dans la discussion, le lien entre la mère et l'enfant est, en revanche, essentiel aux yeux des psychanalystes et pas seulement en raison de son carac- tère structurant normatif. Il l'est avant tout par sa dimension dans la construction de l'identité :

« au-delà du sexe biologique, au-delà du sexe chromosomique même, dans les états intersexuels, l'identité sexuée se fait essentiellement sous la dépendance du rapport parental. » (Green, 1983 : 101)

Autrement dit, pour les psychanalystes, le lien mère-enfant est non seulement partie prenante de la formation des comportements sociaux, il y joue un rôle capital. Aux yeux de Bernard Juillerat - après André Green et Marie-Blanche Tahon (1995) -, la « tache blanche » laissée par Claude Lévi-Strauss sur la relation mère-enfant, c'est celle du biologique, " de la naturalité attribuée aux liens mère/enfant» (Juillerat, 2001 : 122). Or, cette naturalité-là ne date pas d'aujourd'hui, elle est le résultat d'une histoire bien particularisée sur laquelle nous allons brièvement revenir.

\section{Quelle « nature » ?}

L'opposition nature/culture, dans sa version la plus connue aujourd'hui, est sans doute celle que Claude Lévi-Strauss a élaborée pour servir de fondement à sa théorie de la prohibition de l'inceste et de l'alliance. Pour ce dernier, on le sait, la prohibition de l'inceste représente la règle par excellence : ressortissant à la fois à la nature et à la culture, son institution marque le passage de l'état de nature à l'état de société. Telle que la conçoit Lévi-Strauss, la dichotomie nature/ culture est davantage un outil analytique qu'une composante du monde environnant. Quand il oppose la culture à la nature, il n'entend pas, par ces termes, les définitions courantes qu'on en donne habituellement. Le terme de culture désigne, pour lui, l'état de société tel qu'il résulte de l'imposition de la règle, autrement dit de l'interdit de l'inceste. Dans cette perspective, la "nature» fait référence à un état du genre humain, celui qui existait avant que ne soit instaurée la société, c'est-à-dire avant que ne soient élaborées la prohibition de l'inceste et les règles d'exogamie. On se souvient de la célèbre formule :

« La prohibition de l'inceste est, à la fois, au seuil de la culture, dans la culture et, en un sens, [...] la culture elle-même. » (Lévi-Strauss, 1967 : 14)

Cependant, Lévi-Strauss donne cette précision, importante pour notre propos : 
« Envisagée du point de vue le plus général, la prohibition de l'inceste exprime le passage du fait naturel de la consanguinité au fait culturel de l'alliance » (1967 : 35).

Retenons pour l'instant que, pour LéviStrauss, la filiation relève de la nature contrairement au phénomène de l'alliance, « le seul sur lequel la nature n'a pas déjà tout dit »(1967: 36). En réalité, la notion de nature a fait l'objet de spéculations et de discussions bien avant l'élaboration de la théorie de l'alliance de LéviStrauss, au sein du vaste mouvement d'idées neuves qu'on appelle Les Lumières, qui se met en place au XVII ${ }^{\mathrm{e}}$ siècle et s'épanouit au XVIII ${ }^{\mathrm{e}}$. Elle y tient alors un rôle vedette mais avec un sens quelque peu différent de celui que lui attribue Lévi-Strauss aujourd'hui.

Dans un article paru dans l'ouvrage devenu classique de Carole MacCormack et de Marilyn Strathern (1980), Maurice Bloch et Jean H. Bloch font une analyse très fine des « dialectiques de la nature » dans la pensée des philosophes français du XVIII ${ }^{\mathrm{e}}$ siècle (1980 : 25-41). Il existait bien à l'époque une polémique à propos de la nature mais celle-ci concernait l'opposition entre la nature et «quelque chose d'autre », opposition dans laquelle la notion de nature a constitué le moyen de comprendre la société de l'époque et simultanément de la critiquer, à un moment de profonds bouleversements, à la fois matériels, symboliques et idéologiques ${ }^{2}$. Pensée et utilisée par les philosophes français comme une catégorie critique, la « nature » se trouve en quelque sorte revalorisée. Pour Rousseau, par exemple, le concept de «nature » prend sens dans l'opposition à la société de droit divin, injuste et corrompue, et la nature devient la source même de la régénération de la société. Pourtant, au sein de ce mouvement de revalorisation, il demeure un champ de conceptualisation de la nature où ces mêmes philosophes se montrent fort conservateurs : celui où se construit la relation entre la nature et le sexe féminin. En effet, les femmes d'une manière générale étaient considérées par ces derniers comme entretenant un lien puissant avec l'état de nature, en particulier dans ce qui était pensé comme leur domaine, à savoir les procès de la parturition et de l'enfantement. La femme se trouvait plus proche de la nature que l'homme en raison de son rôle physiologique dans la sexualité et plus encore dans l'enfantement (Bloch et Bloch, $1980: 32$ ).

Ces préoccupations nouvelles prennent appui sur les découvertes de la science, en particulier la science médicale et ce qu'elle dit de la physiologie féminine. Rappelons que, selon Thomas Laqueur, c'est précisément à la même période que fut «inventé le sexe tel que nous le connaissons», autrement dit que « prit corps » l'affirmation de la coexistence de deux sexes, non seulement différents mais incommensurables (Laqueur, 1992 : 170). Cette « différence incommensurable » des sexes prend pour fondement le corps et tout particulièrement l'appareil reproductif féminin :

« La matrice, qui avait été une sorte de phallus en négatif, devint l'utérus, c'est-à-dire un organe dont les fibres, les nerfs et la vascularisation offraient une explication et une justification naturalistes du statut social des femmes. » (Laqueur, 1992 : 173)

C'est enfin au sein du même mouvement que se mit progressivement en place le « dispositif de sexualité » décrit et analysé par Michel Foucault, dispositif qui se généralisera au XIX ${ }^{\mathrm{e}}$ siècle (Foucault, $1976: 16)^{3}$.

Au cœur de ces préoccupations concernant la nature, la sexualité, le corps et la physiologie féminine, émergent, assez logiquement, les questionnements sur l'enfantement. Dès le XVIII ${ }^{\mathrm{e}}$ siècle, on assiste d'ailleurs à un glissement lexical qui n'est pas le fruit du hasard: le terme de génération est remplacé par celui de reproduction (Laqueur, 1992 : 177). On voit alors se généraliser cette conception naturalisante des procès liés au corps et à la sexualité : c'est ainsi qu'on attribue un fondement naturel - qui, pour beaucoup de contemporains, tenait lieu d'explication rationnelle - à la hiérarchie des sexes/genres, comme on le fera d'ailleurs, un peu plus tard $\left(\mathrm{XIX}{ }^{\mathrm{e}}\right)$, à celle des « races ». Pour Maurice Bloch et Jean H. Bloch, comme pour Laqueur et Foucault, le recours à la science et à ses découvertes ne rend pas plus « vraies » ces conceptions nouvelles concernant le corps, les sexes et la sexualité. Comme celles dont elles ont pris la place l'idée du sexe unique, par exemple -, elles sont le fruit d'une construction intellectuelle, idéologique ou morale (Bloch et Bloch, Foucault) ou encore politique (Laqueur).

2. Ces considérations sur la nature succèdent aux idées des philosophes de la loi naturelle (XVII ${ }^{\mathrm{e}}$ siècle) - Grotius, Pufendorf, Hobbes et Locke - bâtisseurs de la théorie selon laquelle l'état de société succède à l'état de nature au travers de l'établissement du contrat social (Bloch et Bloch, 1980).

3. Pour l'auteur de l'histoire de la sexualité et contrairement à une idée toute faite fort répandue, ce dispositif-là ne correspondait nullement à la répression sur le sexe des classes à exploiter, il ne s'agissait ni d'un « renoncement au plaisir » ni d'une "disqualification de la chair » mais bien plutôt de l'auto-affirmation d'une classe, au travers d'une manière neuve de considérer et de traiter le corps et le sexe (Foucault, 1976). 
Aujourd'hui, l'attribution du caractère « naturel » à un certain nombre de phénomènes et de comportements sociaux est restée une habitude bien ancrée dans nos sociétés, y compris dans le domaine des sciences humaines et sociales, même si un certain nombre de travaux ont montré depuis plusieurs décennies que notre manière de penser l'opposition nature/culture est historiquement et culturellement marquée. Dans le champ des études féministes de la décennie soixante-dix $\mathrm{du} \mathrm{xx}^{\mathrm{e}}$ siècle, de nombreux auteurs ont critiqué la fascination occidentale pour l'équation entre "nature» et «femme » et tenté de dénaturaliser le sexe, par exemple en s'appropriant la notion de genre 4 . Dans un article précurseur à l'époque, Marilyn Strathern a brillamment déconstruit le raisonnement sur lequel est bâtie une semblable assertion et a montré qu'il s'agit davantage d'une représentation/croyance que d'une véritable connaissance, fondée scientifiquement (1980: 183). En France, la réflexion sur l'assignation des femmes à des rôles "naturels » et ses conséquences - aux plans épistémologique, éthique, politique - se développe aussi avec les travaux de Françoise Héritier (1981) et de Nicole-Claude Mathieu (1991) 5 .

Mais, si on accepte bien plus volontiers aujourd'hui de considérer que ce que nous appelons ordinairement "nature » peut être représenté dans certaines sociétés comme un élément constitutif de la culture, pour le dire dans les termes de Philippe Descola (1986 : 401), il reste beaucoup plus difficile de se débarrasser de cette même habitude de penser quand il s'agit de procès associés aux fonctions féminines et plus particulièrement à ce qui demeure considéré comme la fonction féminine par excellence, celle de l'enfantement (voir aussi Descola, 2005).

Selon le dictionnaire Larousse, le terme « engendrer» signifie « reproduire par génération, procréer ». Cependant, Le Robert (1964) donne une précision supplémentaire qui ne manque pas d'intérêt : " produire par voie de génération, plus spécialement en parlant du mâle» (1964 : 1584). En parlant de la femme, en effet, on dira plus volontiers " concevoir», ou « enfanter» (1964: 1584). Ici, on utilisera le terme « engendrement » pour parler de la pro- duction par voie de génération quand la distinction de sexe n'est pas pertinente, en réservant le mot « enfantement» à la mise au monde d'un enfant par une femme.

Dans les sociétés de Polynésie, l'opposition nature/culture telle que nous la connaissons n'a pas cours, comme on va le voir. Mais surtout, l'engendrement (quand on parle du père), l'enfantement (quand on parle de la mère) et la naissance (quand on parle de l'enfant) constituent pour les gens concernés un événement hautement «culturel », si on donne à ce terme un sens proche de celui que Claude Lévi-Strauss a donné au mot « culture » dans les Structures élémentaires de la parenté, à savoir un événement qui est l'objet de règles et de normes, de représentations et de valeurs, bref un événement qui « fait société ». On commencera par examiner la manière dont la société tongienne concevait ces étapes du cycle de la vie - accouchement et naissance mais aussi fécondation et gestation - au travers des récits et des mythes de la tradition orale ainsi que des pratiques, rituelles ou non, qui leurs sont associées. Pour ma démonstration, je vais prendre appui sur un ensemble mytho-rituel polynésien où il est abondamment question d'engendrement/enfantement et de naissance, et revenir sur certaines des analyses que j'en ai faites ailleurs. Il s'agit d'une part du mythe d'origine tongien qui relate la création de l'univers et la naissance des grands dieux, d'autre part d'une ablution rituelle prise par une aristocrate de haut rang, la propre sœur du Tu'i Tonga, chef suprême de la société tongienne classique, au moment de l'intronisation de ce dernier ${ }^{6}$. Pour terminer, j'aimerais évoquer un mythe d'origine issu d'une autre société que Tonga mais qui est néanmoins polynésien puisqu'il est issu du corpus de Mangareva (archipel des Gambier). Si je prends la liberté de citer ce mythe qui raconte comment les femmes de cette société ont " appris " à accoucher, c'est dans l'intention de montrer qu'à l'autre bout de la Polynésie, les mythes soulignent à leur tour la dimension éminemment sociale de l'enfantement. Mais revenons d'abord à Tonga où, pour contextualiser le mythe de création, nous allons indiquer brièvement ce que les pratiques rituelles et/ou quotidiennes disent des procès associés à l'enfantement.

4. Cette double analogie homme-culture/femme-nature avait fait l'objet d'un article retentissant écrit par Sherry Ortner dans un volume fondateur de l'anthropologie féministe dirigé par Michelle Zimbalist Rosaldo et Louise Lamphere (1974). À propos de la dénaturalisation du sexe, de l'appropriation féministe de la notion de genre et de son ambiguité fondatrice, voir Éric Fassin (2008: 75-392).

5. À la suite du travail critique précurseur de Simone de Beauvoir sur le sujet (1949).

6. Ce geste rituel singulier, propre à la société classique des îles Tonga ( $\mathrm{XIX}^{\mathrm{e}}$ siècle), relevé par le voyageur Dumont d'Urville, paraît avoir échappé à l'attention des exégètes, pourtant nombreux et perspicaces, de l'histoire tongienne. Il s'agit d'une ablution rituelle, prise par une femme d'un rang particulièrement exalté, la Tu'i Tonga Fefine, sœur du souverain de Tonga, le Tu'i Tonga, dans un contexte rien moins qu'anodin, celui de l'intronisation d'un nouveau Tu'i Tonga (Douaire-Marsaudon, 2002). 


\section{Fécondation, gestation, naissance}

L'univers mythique de ces sociétés est saturé d'engendrements. D'une manière générale, les héroïnes des récits de la tradition orale sont présentées comme devant parvenir à cet accomplissement : la mise au monde d'un enfant (fanau). Dans le Tonga d'avant la christianisation, l'enfant était conçu comme un don des esprit ancestraux à la société des «vivants» (les humains) ${ }^{7}$. Dans la tradition orale, un certain nombre de fécondations ne résultent pas d'un coït entre un homme et une femme mais d'un phénomène d'absorption ou d'imprégnation du corps féminin par une substance fécondante d'origine divine (Douaire-Marsaudon, 2002: 519-520) ${ }^{8}$. D'autres récits rapportent des parthénogenèses féminines, auxquelles les exemples précédents peuvent être assimilés puisque les fécondations impliquées ne sont pas le résultat d'une copulation avec un partenaire masculin. Quelques-uns mettent en scène la manière dont certaines denrées du monde divin - l'igname réservée aux chefs, par exemple - ont été volées aux divinités et mises à la portée des humains par une femme, plus précisément par l'intermédiaire du corps féminin (dans le cas de l'igname, une femme le cache dans son vagin et en accouche, une fois revenue sur terre (à Tonga). Tous ces récits exaltent les surprenantes capacités génésiques du corps féminin, médiateur entre l'au-delà divin et le monde des «vivants 》 (DouaireMarsaudon, 1998 : 185), mais ils sont aussi chargés de signifier la prodigieuse puissance féconde des ancêtres et des divinités.

À Tonga, dans la société du début du $\mathrm{XIX}^{\mathrm{e}}$ siècle où ces récits avaient cours, tout ce qui entoure la gestation et surtout la naissance était censé relever d'un secret dont les femmes - les mères, les tantes maternelles et les grands-mères - avaient la charge exclusive (Martin, 1981: 461) et dont elles se transmettaient les connaissances de génération en génération. Le père participait à la croissance de son enfant in utero en nourrissant le placenta - fonua ou «pays » du fœtus c'est-à-dire en procurant à la mère de la nourriture produite et cuite par lui. Cependant il existait au sein de la famille un personnage-clé, à qui revenait le premier rôle au cours de la gestation et de la naissance d'un enfant : la sœur du père ou mehekitanga. Cette dernière était censée pou- voir jeter des sorts à sa belle-sœur enceinte, provoquer une fausse-couche ou un accouchement avant terme ; un enfant mort-né ou une naissance prématurée entraînant un handicap étaient mis d'abord au compte de la tante paternelle ${ }^{9}$. $\mathrm{Si}$, au moment précis de la naissance, la mehekitanga n'est pas admise dans l'entourage proche de la nouvelle accouchée, elle occupe cependant la place d'honneur dans le rituel qui consacre l'arrivée de l'enfant dans le groupe (kainga) paternel. En signe qu'elle accepte le nouveau venu dans ce groupe qu'elle est chargée de représenter, elle donne à la mère du bébé un présent, une sorte de « lit» (pae) constitué d'un morceau de tissu d'écorce recouvert d'une natte fine (kie). Au cours de la cérémonie, elle occupe la place d'honneur et elle reçoit ou prélève la plus belle part - natte et tissu d'écorce - de tous les dons, ou « richesses » (koloa) apportées par les deux clans, paternel et maternel. Au terme de la cérémonie, c'est elle qui lève les tabous mettant fin à la période très critique que représente l'accouchement. Celui-ci, en effet, est considéré comme une période très singulière - comme l'est aussi un décès - en raison de l'effacement momentané des limites séparant l'au-delà des morts et des divinités du monde des " vivants » (les humains), source de danger pour ces derniers. Le terme de référence utilisé par la tante paternelle pour nommer ses neveux (les enfants de frère) est celui de fakafotu: de faka, "à la manière de » et de fotu, "faire apparaître » ou " faire fructifier ». Au nom des ancêtres, la tante paternelle est celle qui accepte les enfants de ses frères dans le clan paternel, celle qui les fait apparaître et fructifier. C'est bien leur mère qui les a mis au monde mais c'est leur tante paternelle qui leur accorde de vivre, de s'intégrer dans la société et de donner naissance à leur tour à une lignée. Les plus hautes marques de respect et de soumission sont dues à la tante paternelle et la transgression de cette règle peut entraîner malaise, maladie ou même mort.

Cependant, au sein de la parenté étendue, une autre figure parentale apparaît comme le pendant de la tante paternelle, son double inversé : l'oncle maternel. Ces positions symétriques inversées découlent elles-mêmes des normes et valeurs imposées à la relation frère/sœur, fondatrice de l'ordre parental. Tout homme étant d'un rang inférieur à toutes ses sœurs (quel que soit

7. Seuls les esprits ancestraux étaient censés maîtriser les forces de vie (mana) et autoriser l'arrivée d'un nouveau venu au sein du clan familial. Aujourd'hui, la naissance d'un enfant est certes attribuée au dieu chrétien mais l'idée de la volonté des ancêtres dans la conception et la gestation d'un enfant est toujours vivace.

8. Telle femme est fécondée par le rayonnement solaire, en présentant ses fesses au vent ou encore en se baignant dans le bassin d'un dieu-serpent.

9. Ces représentations attachées au rôle de la tante paternelle n'ont pas disparu. 
l'âge de celles-ci), il est aussi considéré comme d'un rang inférieur aux enfants de ses sœurs et ses propres enfants sont eux-mêmes d'un rang inférieur à leur tante paternelle et aux enfants de cette dernière. Il en résulte que l'oncle maternel est la personne de la parentèle avec laquelle l'on peut entretenir la relation la plus libre, la plus dénuée de tabou. On peut parler et rire d'à peu près tout avec son oncle maternel, on peut se montrer à lui dans une tenue négligée, on peut le toucher ou terminer ses plats ou se saisir de ses habits, sans crainte d'une sanction surnaturelle. L'oncle est censé prendre soin des ses neveux utérins, les conseiller et les guider tout au long de leur vie. Surtout, ses neveux lui étant supérieurs en rang, ils accèdent librement non seulement à sa personne mais à ses biens. Les voyageurs européens ou les missionnaires de l'époque du contact ont relaté avec étonnement les exactions des ces neveux utérins, en particulier au sein de la chefferie de haut rang (Douaire-Marsaudon, $1998: 233 s q$ ). Aujourd'hui, dans un pays marqué par la soif de terres, il est devenu courant que les neveux utérins demandent à leur oncle maternel de pouvoir cultiver un morceau de terre pris sur son domaine. C'est ce que l'on appelle les droits et privilèges du $f a h u$, équivalent tongien de l'institution bien connue du vasu à Fidji.

$\mathrm{Au}$ sein des rituels concernant ses neveux, l'oncle maternel occupe une place inversée par rapport à celle de la tante paternelle : lors du décès d'un enfant de sœur, il demeure près du four, où son bas statut l'autorise seulement à préparer les victuailles pour les invités. Surtout, au moment de l'accouchement de sa sœur, il doit maintenir au chaud les jeunes noix de coco (veifua), censées faire monter et accroître le lait (de sa sœur) destiné au nouveau-né. Il est là typiquement dans son rôle de fa'e tangata, terme de référence qui est le sien et qui signifie, littéralement, « mère masculine $»^{10}$.

Ces rôles, symétriques et inverses, de la sœur $\mathrm{du}$ père et du frère de la mère montrent le poids de la relation frère-sœur au sein de la parenté tongienne mais ils soulignent aussi l'importance de la distinction filiative - pour emprunter ses termes à Bernard Juillerat - dans la formation du sujet : ici, les figures parentales sont en quelque sorte redoublées et recomposées par leurs germains de sexe opposé. Nous allons maintenant présenter le mythe tongien de création du monde.

\section{Un évolutionnisme polynésien}

Comme souvent dans les cosmogonies polynésiennes, le mythe d'origine tongien inscrit la genèse du monde, des dieux et de l'humanité dans une structure généalogique :

« Le Limu (plante marine) et la Kele (vase), attachés ensemble et ballotés par les flots, abordent au Pulotu et $\mathrm{y}$ enfantent une pierre ferrugineuse qu'ils appellent Touiafutuna. La pierre ferrugineuse se met à trembler et à gronder, puis elle se fend à quatre reprises et donne naissance à quatre paires de jumeaux de sexe opposé. La première paire de jumeaux s'unit et enfante à son tour deux jumeaux de sexe opposé11 : un garçon, Taufulifonua, et une fille, Havealolofonua. La deuxième et la troisième paire de jumeaux enfantent chacune une fille, Velelahi et Velesii. Une fois devenue grande, Havealolofonua, fille des premiers jumeaux, s'en va trouver ses "sœurs" cadettes et leur dit que leur frère étant le seul être masculin, elles doivent toutes s'unir à lui. De ces trois dernières unions naissent alors les grands dieux : de la sœur aînée, Hikuleo ; de la sœur cadette, les Tangaloa; et, enfin, de la benjamine, les Maui. À Hikuleo, parce qu'il est né de la sœur aînée, revient la tâche de partager le monde : aux Tangaloa et à leur mère, il attribue le ciel, le Langi ; aux Maui et à leur mère, il attribue le monde souterrain, le Lolofonua ; il régnera lui sur le Pulotu, le paradis tongien où s'en reviennent les âmes des chefs après leur mort. Afin de peupler son empire, Hikuleo cherche d'abord à faire périr tous les premiers-nés. Aussi, ses frères, les Tangaloa et les Maui, décident-ils de l'attacher avec une corde dont l'un des bouts est accroché par les Tangaloa au ciel et l'autre par les Maui à la terre. Désormais, Hikuleo promène son regard sur la terre et les mouvements qu'il fait pour se détacher provoquent les tremblements de terre. » (d'après Reiter, 1907 : 230-240)

Ce mythe nous fait assister à une suite d'événements qui prennent place dans une chronologie évolutive constituée d'épisodes successifs que l'on peut identifier comme suit :

1. un accouplement primordial (première génération) au paradis, ce qui donne :

2. la naissance d'une pierre (deuxième génération) ;

3. la pierre enfante (fanau) quatre paires de jumeaux (troisième génération) dont trois s'accouplent, ce qui donne :

10. Lorsque la circoncision se pratiquait dans le cercle familial - et non à l'hôpital comme aujourd'hui -, c'était l'oncle maternel qui se chargeait de l'opération sur ses neveux utérins. Lui seul, en effet, pouvait faire couler le sang de ces derniers, puisque, selon la théorie de la transmission du sang, un homme partage le même sang avec ses sœurs et avec les enfants de ces dernières (mais il ne partage pas le même sang avec les enfants de ses frères ou avec ses propres enfants).

11. Dans le mythe rapporté par le père Reiter (1907), Taufulifonua et Havealotofonua sont présentés comme frère et sœur, sans que leur gémellité soit précisée. Cependant, dans d'autres mythes où ils figurent, ils sont présentés comme des twin deities, des jumeaux divins (Gifford, 1924 : 14). Selon la tradition orale, ils sont les initiateurs des premiers rapports sexuels (ibidem). 
4. trois naissances anthropoïdes dont l'une est gémellaire : un garçon et trois filles (quatrième génération), lesquelles s'accouplent avec leur « frère » ce qui donne :

5. la naissance des grands dieux : les quatre Tangaloa, les trois Maui et Hikuleo (cinquième génération).

La fin du mythe est centrée sur la figure d'Hikuleo et sur ses rapports avec ses frères :

- Hikuleo fait le partage du monde : aux Tangaloa et à leur mère, le ciel; aux Maui et à leur mère, l'intérieur de la Terre ; à lui-même, le paradis tongien.

- Hikuleo veut faire mourir l'espèce humaine mais ses frères l'attachent au centre du cosmos.

On a ici, typiquement, ce que Dixon a appelé un mythe " évolutionnaire » où l'on passe d'un monde végétal et minéral (la vase, l'algue, la pierre) à un monde animal (tortues, anguilles, pigeon) avant de parvenir à l'âge anthropomorphique, réalisé à la quatrième génération, puis à l'organisation et à la mise en ordre du cosmos, accomplies par les grands dieux, Hikuleo d'abord, ses "frères » ensuite. Il y a bien ici un schéma général qui sous-tend une idée/représentation de l'histoire de l'humanité, laquelle évolue lentement à partir d'un monde préexistant, englobant et antérieur, constitué par l'océan et dont le point central est le Pulotu, le lieu des grands commencements, là où se font les engendrements menant à la métamorphose du monde et à la création/naissance des grands dieux. Celle-ci est précédée par la séquence de l'inceste « généralisé » où l'unique frère s'unit à ses « sœurs », sa jumelle et ses deux cousines, unions gémellaires incestueuses d'où sont issus Hikuleo, les Tangaloa et les Maui.

\section{Naissance des grands dieux et mise en ordre du cosmos}

Les Tangaloa et les Maui sont des divinités communes à l'ensemble du monde polynésien. Tangaloa est associé le plus souvent à la mer, parfois au ciel, et couronne le panthéon divin dans nombre de cosmogonies. Maui est plutôt un demi-dieu ou un héros qu'une divinité à part entière. Puissance chtonienne associée au Lolofonua, autrement dit à l'intérieur de la Terre, Maui est aussi le type même du trickster, héros civilisateur qui vole aux grands dieux les biens nécessaires aux hommes ${ }^{12}$. À Tonga, les Tangaloa sont au nombre de quatre, chacun d'eux correspondant à l'un des statuts majeurs de la pyramide sociale. L'aîné des Tangaloa, Eitu' matupu'a (littéralement, l'esprit ancêtre), associé au ciel, est le père de 'Aho'eitu (littéralement, l'esprit jour), le premier Tu'i Tonga, chef suprême de la société tongienne ${ }^{13}$. Quant aux Maui, les Tongiens se les représentent sous la forme d'une triade composée de trois frères ou, plus souvent, d'un grand-père, d'un père et d'un fils/petit-fils. Autrement dit, les Maui apparaissent comme la métaphore même de la filiation et de la succession des générations.

En ce qui concerne Hikuleo, il est clairement identifié comme l'aîné des dieux ; cette place lui est dévolue, dit le mythe, parce qu'il est le fils aîné de la fille aînée. Fort de cette préséance, Hikuleo fait le partage du monde : aux Tangaloa et à leur mère, il donne le ciel, aux Maui et à leur mère, la Terre; il s'attribue le Pulotu, le lieu-origine de la création, lequel est aussi le paradis tongien où sont accueillis les esprits des chefs après leur mort et où coule le Vaiola, source qui redonne vie, jeunesse et beauté. Les Maui deviennent les divinités du Lolofonua (l'intérieur de la terre) parce qu'ils y sont envoyés "avec leur mère ", comme les Tangaloa deviennent les divinités du ciel parce qu'ils s'y installent avec la leur. Dans le mythe, c'est donc le lien de filiation utérine qui fonde le rapport originaire des grands dieux à leurs domaines cosmiques respectifs - le ciel, la terre - exactement comme, dans le monde humain, le lien à la mère, plus exactement au groupe de la mère (kainga i'fa'e) fonde l'autochtonie. Lors d'une naissance, dans la société classique comme encore aujourd'hui, le placenta, puis le cordon ombilical du bébé, lorsqu'il tombe, sont enterrés sur les terres familiales du groupe maternel, marquant ainsi l'attache de chacun à son groupe/lieu d'origine ${ }^{14}$.

12. Maui Kisikisi (le petit) est crédité de travaux prométhéens : à Tonga, la pêche des îles, le soulèvement du couvercle du ciel, la régularisation des mouvements du soleil, le vol du feu souterrain, la domestication de la poule et du chien, tous deux géants et mangeurs d'homme, ainsi que le mûrier à papier (Broussonetia papyrifera), lui aussi carnivore et qui sert à faire le papier d'écorce si important dans la vie quotidienne et rituelle (Williamson, $1933: 217 s q$ ).

13. Un long mythe raconte la « double » naissance de 'Aho'eitu : né des amours de Eitu'matupu'a et d'une fille de chef, il monte au ciel pour rencontrer son père où il est assassiné et dévoré par ses frères célestes, avant d'être ressuscité par son père et envoyé par ce dernier pour régner sur terre (Tonga) (Douaire-Marsaudon, $2002: 522$ ).

14. Les femmes tongiennes avaient l'habitude de retourner dans leur groupe de naissance pour accoucher. L'importance du symbolisme associé au traitement du placenta et du cordon ombilical est attestée dans nombre de sociétés de Polynésie, au point que l'anthropologue Bruno Saura a pu parler de «culture placentaire » $(2008: 162)$. 
Si Hikuleo possède la préséance qui lui permet de présider à la mise en ordre de l'univers sociocosmique, le mythe l'oppose pourtant à ses frères, et ceci à deux plans différents ${ }^{15}$. D'une part, ces derniers sont obligés de l'attacher au centre de l'univers, grâce à une corde dont ils retiennent les extrémités, de peur qu'il ne détruise les premiers-nés des humains, les chefs, dans la conception polynésienne ${ }^{16}$. D'autre part, alors que les autres divinités sont multiples - les Tangaloa sont quatre et les Maui trois - Hikuleo, en revanche, paraît être unique; par ailleurs, de tous les grands dieux, il est le seul à ne pas avoir de descendance. Dans les récits de la tradition orale, le personnage d'Hikuleo est toujours représenté comme un dieu terrifiant, une divinité de la démesure, qui possède de nombreux aspects duels. On peut gager qu'il est une sorte de " deux-en-un », ses attributs constituant autant de manifestations de sa nature bisexuée (Douaire-Marsaudon, 1998 : 283).

Il paraît à peu près certain qu'à l'époque du contact en tout cas et probablement bien avant, on ne rendait aucun culte ni aux Tangaloa, ni aux Maui. En revanche, c'est en l'honneur d'Hikuleo qu'avait lieu, chaque année, le 'inasi, le plus grand et le plus important des rites de la vie religieuse tongienne. Au cours de ce rite, qui durait une dizaine de jours, c'est l'ensemble des Tongiens qui offraient à leurs souverains les prémices des récoltes, maritimes ou terrestres ${ }^{17}$. À cette occasion, des quantités considérables de nourritures (porcs, ignames, patates douces, poissons, noix de coco, etc.) et de "richesses" (nattes et tissus d'écorce) étaient rassemblées selon l'ordre hiérarchique et accumulées sur le mala'e royal avant d'être finalement redistribuées. Cependant, avant que ne commence la redistribution, le Tu'i Tonga prélevait la plus belle part des produits accumulés et les offrait à sa sœur, la Tu'i Tonga Fefine qui se trouvait ainsi « on the receiving end of the tribute» (Bott, 1982 : 107). Selon mon hypothèse, ce n'était pas uniquement le chef suprême, le Tu'i Tonga, qui était la personnification de Hikuleo au moment du rituel du 'inasi, mais lui et sa sœur, la Tu'i
Tonga Fefine ${ }^{18}$. Dans et par le rituel, ils sont l'un et l'autre les représentants terrestres de la divinité, sectionnée, divisée en ses deux pôles sexués sous la forme d'un frère et d'une sœur, à qui l'inceste est interdit : ainsi pourra vivre et croître la société des « vivants » (humains).

Hikuleo, condensé d'inceste frère/sœur dont la figure cosmique est attachée au centre même de l'univers, est donc bien une allégorie à double détente symbolique. Né(e) de l'unique homme du temps des grands commencements et de sa seule " vraie » sœur, maître du monde des origines, le Pulotu, où coule le fleuve qui redonne vie et beauté, Hikuleo veut faire mourir les " vivants" afin de peupler son royaume mais il est aussi la divinité pourvoyeuse de la fertilité, terrestre et maritime en l'honneur de laquelle avait lieu le gigantesque rituel propitiatoire du 'inasi. Personnage paradoxal donc que cet Hikuleo bifrons, divinité mortifère et stérile, contrôlant pourtant la source du retour à la vie et présidant aux arcanes de la fertilité générale.

Le mythe d'origine tongien raconte comment l'univers socio-cosmique a pris forme au travers d'une série d'accouplements et d'enfantements qui donne naissance aux grands dieux. Dans la mesure où l'inceste est ici la règle, on pourrait dire, en appliquant stricto sensu le terme de " nature » tel qu'il est conçu par Lévi-Strauss, qu'on est là dans un monde " naturel », infrasocial en quelque sorte. Reste à savoir si le mythe et plus largement l'imaginaire tongien raisonnent de cette manière.

Nous allons quitter le mythe d'origine tongien et présenter un geste rituel singulier, celui de l'ablution prise par la personne qui possède le rang le plus exalté de toute la société tongienne, la sœur du Tu'i Tonga, la Tu'i Tonga Fefine (littéralement, Tu’i Tonga femme).

\section{Le bain sacré de la Tu’i Tonga Fefine}

La mention du bain de la princesse est contenue dans les relations de voyage de Dumont $\mathrm{d}^{\prime}$ Urville $^{19}$. Le navigateur livre un détail fourni par ses informateurs à propos de l'intronisation

15. Le mythe fait d'Hikuleo la figure dominante des dieux, alors que dans la plupart des archipels polynésiens-sauf à Samoa -, c'est à Tangaloa (Tagaloa, Ta'aroa, etc.) à qui est généralement attribuée cette place. On peut penser que la figure d'Hikuleo, dont le Tu'i Tonga était considéré comme le grand prêtre, a pris la place de Tangaloa dans ces deux sociétés au cours d'une histoire qui a vu le renforcement progressif du pouvoir (pourtant souvent contesté) des Tu'i Tonga.

16. Comme on ne conçoit pas la société sans chef, ce que menace Hikuleo, c'est l'ensemble de la société des «vivants », autrement dit des hommes.

17. La présentation des prémices était suivie de concours de danses et de matchs sportifs ; le dernier jour se clôturait sur les fameuses danses de nuit célébrant la fécondité générale.

18. Les commentateurs du 'inasi (Gifford, 1929; Urbanowicz, 1972), en s'appuyant sur le témoignage de Sarah Farmer (1855), mentionnent simplement que, lors du rituel, le Tu'i Tonga est le représentant ou le grand prêtre de Hikuléo.

19. «Cet arbre gigantesque est particulièrement dédié au toui-tonga. Immédiatement après son couronnement, ce dignitaire vient se placer sous l'ombrage de ce mea. Là, sur un siège préparé à cet effet, et entouré de ses officiers, il accomplit certaines 
du chef suprême de la société tongienne, le Tu'i Tonga. Ce rituel, qui se déroulait après un long deuil de six mois consécutif à la mort du précédent chef suprême, avait un caractère d'une extrême solennité. Pendant que le futur Tu'i Tonga accomplit « certaines cérémonies », nous dit Dumont d'Urville, la «toui-tonga Fafine» (la Tu'i Tonga Fefine) va se purifier dans une fontaine voisine, entourée de ses femmes. Nul homme, précise-t-il, ne peut se baigner dans cette fontaine sous peine de mort (Dumont d'Urville, 1833, t. I : 109).

Précisons d'abord que, lors de l'intronisation d'un nouveau Tu'i Tonga, la Tu'i Tonga Fefine en titre est la sœur du Tu'i Tonga décédé et par conséquent la tante paternelle de celui que l'on s'apprête à revêtir des insignes de sa fonction (Douaire-Marsaudon, 1998 : 228). Ce qui intrigue, dans cette ablution royale, n'est pas tant son existence en soi, ni même le fait qu'elle soit assujettie à des tabous sévères, mais son occurrence dans le contexte tout à fait singulier de l'intronisation du chef suprême, rite d'une dimension, politique et symbolique, considérable. Pourquoi cette ablution rituelle prise par une personne qui, après tout, n'est qu'une parente du héros du jour, le nouveau Tu'i Tonga? Et de quoi cette parente est-elle donc censée se purifier ?

Dans la société tongienne du $\mathrm{XIX}^{\mathrm{e}}$ siècle, les femmes avaient recours à une ablution rituelle purificatrice dans une occasion bien particulière, quand elles venaient d'accoucher ${ }^{20}$. Cette ablution visait à mettre fin à la période des tabous pesant sur la parturiente et à la réintégrer, après ce moment de liminalité intense que constituait l'accouchement, dans le monde ordinaire des « vivants ». Le bain de la Tu'i Tonga Fefine au moment même de l'intronisation du Tu'i Tonga paraît donc suggérer une "mise au monde » symbolique, qui ne peut être que celle du nouveau Tu'i Tonga par la Tu'i Tonga Fefine.

On sait que les rituels d'intronisation prennent souvent la forme d'une réplique des rituels de naissance ${ }^{21}$. Le futur souverain est investi de sa fonction non pas en « recevant son office » mais en «naissant au monde » qu'il doit gouverner, revêtu ontologiquement, pourrait-on dire, de ses attributs. Mais si le bain rituel de la Tu'i Tonga Fefine sert à signifier qu'elle vient d'accoucher symboliquement du nouveau Tu'i Tonga, une telle naissance pose à son tour question. Pourquoi le nouveau souverain est-il censé (re)naître de sa tante paternelle ? Que manque-t-il donc à la première naissance du Tu'i Tonga pour que soit signifié, par une véritable mise en scène cérémonielle, qu'il naît au monde à nouveau, de la Tu'i Tonga Fefine cette fois ? Par ailleurs, ce que le bain sacré de la grande dame paraît aussi - et par voie de conséquence - suggérer, c'est l'existence en amont de l'intronisation royale d'une union entre la Tu'i Tonga Fefine et le père décédé du récent intronisé, autrement dit, entre l'éminente princesse et son frère. On sait que certaines sociétés (Égypte pharaonique, Pérou incaïque, Hawa'i) pratiquaient le mariage hiérogamique entre frère et sœur au plus haut niveau de l'ordre socio-hiérarchique, autorisant ainsi ceux qui le couronnaient à agir à l'instar des divinités, en transgressant certaines de ses lois fondatrices. Soit ! Mais à Tonga, le Tu'i Tonga n'épousait pas la Tu'i Tonga Fefine. Même si la tradition orale tongienne rapporte un inceste entre la première Tu'i Tonga Fefine et son frère le Tu'i Tonga, l'étude des généalogies royales montre clairement que la Tu'i Tonga Fefine n'était pas censée devenir l'épouse du Tu'i Tonga, ni a fortiori donner naissance à l'héritier au titre ${ }^{22}$. D'une manière générale, dans la société tongienne - à l'âge classique comme aujourd'hui -, la relation qui lie un frère et une sœur est marquée par un puissant tabou qui marque de l'empreinte de l'interdit tout ce qui relève de la sexualité23. Pourtant, l'ablution rituelle de l'éminente princesse paraît bien renvoyer à l'idée d'une union hiérogamique frère/sœur, laquelle serait en quelque sorte fantasmée à la fois dans le mythe (l'inceste entre la première Tu'i Tonga Fefine et son frère) et dans le rite.

cérémonies, tandis que la toui-tonga Fafine va se purifier dans une fontaine voisine assistée de quatre ou cinq femmes. Aucun homme ne peut se baigner dans cette source sous peine de mort. » (Dumont d'Urville, 1833, t. I : 109).

20. C'est aussi le cas dans la société contemporaine.

21. C'était notamment le cas à Hawai'i pour l'intronisation de certains chefs de haut rang où on rejouait symboliquement la cérémonie de la coupure du cordon ombilical ainsi que le rituel de circoncision (Kamakau, 1991 : 54 ; voir aussi Sahlins, 1985 : 212).

22. La tradition orale rapporte que la Tu'i Tonga Fefine demeura longtemps interdite de mariage, en raison de son rang très sacré. À partir du XVII e siècle, elle fut finalement autorisée à épouser un prince «étranger », un descendant de la Fale Fisi ou maison royale fidjienne, mariage qui se répéta régulièrement jusqu'après l'arrivée des Européens. Auparavant, l'éminente princesse, fille et sœur de souverain, était donc parthénos, c'est-à-dire célibataire mais pouvait néanmoins être mère. Ses rejetons, considérés comme issus d'engendrements divins, étaient eux-mêmes conçus comme trop sacrés pour régner. À partir du mariage de la Tu'i Tonga Fefine, l'habitude fut prise de marier le Tu'i Tonga avec la fille de la lignée royale cadette, mariage répété lui aussi sur plusieurs générations jusqu'au XIX ${ }^{\mathrm{e}}$ siècle (Douaire-Marsaudon, 2002 : 147-162 et 519-528).

23. Entre frère et sœur, il est interdit de faire une quelconque allusion, en paroles ou en gestes, à tout qui concerne la sexualité (ou tout ce qui touche à la sexualité) comme, par exemple, l'accouchement (risque d'évoquer les organes génitaux). 
Pour éclairer le mystère du bain de la Tu'i Tonga Fefine, j'avais proposé une analyse de la souveraineté tongienne à partir de ses principes d'organisation et de l'histoire de ses pratiques ${ }^{24}$. Puisque cette ablution rituelle évoque un accouchement et une naissance, je reprends ici une partie de cette démonstration.

On sait que le $\mathrm{XVII}^{\mathrm{e}}$ siècle tongien a correspondu à une période de changements historiques importants, les souverains suprêmes de Tonga ayant repris en main les rênes du pouvoir après avoir été chassés de l'archipel pendant près d'un siècle. L'un d'entre eux, Tele'a, que la tradition orale présente comme l'un des plus puissants Tu'i Tonga, entreprit un certain nombre de réformes et mit en œuvre une nouvelle politique d'alliance entre les lignées royales en décidant de marier la Tu'i Tonga Fefine. Cette nouvelle situation s'est accompagnée d'un renouveau rituel d'une ampleur certaine, celui-ci étant l'occasion de réaffirmer la part éminemment sacrée de la personne des chefs suprêmes ${ }^{25}$. Il n'est alors pas impossible de considérer que le rite d'intronisation du souverain suprême et, par conséquent, le bain cérémoniel de la Tu'i Tonga Fefine qui en est l'une des pièces maîtresses, auraient tous deux constitué l'un des volets majeurs de cette réforme rituelle. En effet, le bain sacré de la grande dame au cours du rituel d'intronisation du nouveau souverain suprême transformait ce dernier en un être très sacré et parfaitement singulier comme on va le voir maintenant.

\section{La (re-)sacralisation du chef suprême}

Le bain rituel de la Tu'i Tonga Fefine fait du nouveau Tu'i Tonga un être né deux fois ${ }^{26}$. Le nouveau chef suprême est certes né des amours de son père, le précédent Tu'i Tonga, un hommedieu, avec la moheofo, une aristocrate d'un rang tout à fait éminent. Mais il ajoute quelque chose de fondamental à cette première naissance, prestigieuse certes, mais néanmoins passablement « terrestre ». Par la vertu de cet élément symbolique fondamental que représente l'ablution de la grande dame, l'intronisation du nouveau Tu'i Tonga fait de lui, au moment précis où il « naît » à sa fonction, un être « issu » de la Tu'i Tonga Fefine, autrement dit un être unique, engendré humainement certes mais néanmoins revêtu ontologiquement, grâce à sa seconde naissance, de la sacralité ancestrale. Simultanément, en raison du principe de la transmission du rang/sang, ce deuxième engendrement par la Tu'i Tonga Fefine lui procure le rang suprême de sa mère symbolique ${ }^{27}$.

Renaissance rituelle par laquelle le Tu'i Tonga réaffirme la part sacrée de sa nature et surélève son rang, l'ablution de la Tu'i Tonga Fefine réactive aussi les principes cruciaux de la reproduction du groupe de parenté et, par là, ceux de la société toute entière. Rappelons que la Tu'i Tonga Fefine est, au moment de l'intronisation d'un nouveau Tu'i Tonga, la tante paternelle de ce dernier, sa terrible et nécessaire mehekitanga. Par le rite de l'ablution, elle est celle qui, conformément à sa vocation, le fait «apparaître » et «fructifier», non pas comme simple être « vivant »- cela fut le cas à sa naissance - mais comme souverain sacré, tout baigné de l'assentiment des prestigieux ancêtres qu'elle représente. Triple légitimité donc pour le nouvel intronisé par cette re-naissance rituelle qui lui garantit tout à la fois son lien avec l'ordre surnaturel, son rang exalté d'enfant sacré et la bienveillance agissante des divinités ancestrales.

\section{L'inceste sacré des origines}

Reste l'épineuse question de l'inceste royal que soulève, très logiquement, cette deuxième naissance. Car si le nouveau souverain, fils et héritier du Tu'i Tonga précédent, est, par l'ablution de la Tu'i Tonga Fefine, sa tante paternelle, symboliquement né d'elle, alors on ne voit pas comment on pourrait ignorer ce qui, dans cette intronisation sur le mode de la naissance, fait signe, à savoir l'inceste hiérogamique fantasmé entre la Tu'i Tonga Fefine et le précédent Tu'i Tonga, son frère.

24. J'avais eu recours en particulier à l'étude des stratégies matrimoniales qui se mettent en place au XVII ${ }^{\mathrm{e}}$ siècle - et qui perdurent jusqu'à l'époque historique du contact (fin $\mathrm{XVIII}^{\mathrm{e}}$-début XIX ${ }^{\mathrm{e}}$ ).

25. Ce n'est sûrement pas un hasard si c'est précisément au cours du règne du Tu'i Tonga Tele'a, artisan de ces réformes et du mariage de la Tu'i Tonga Fefine, que fut bâti le plus grand et le plus élaboré des mausolées royaux, le pa'epa'e'o Tele'a.

26. Exactement comme son ancêtre 'Aho'eitu, le premier Tu'i Tonga, d'après le mythe d'origine du premier Tu'i Tonga (Douaire-Marsaudon, $2002: 522$ ).

27. À Tonga, il existait deux théories de la transmission des substances. L'une, probablement ancienne - on la trouve ailleurs en Polynésie -, selon laquelle le père et la mère transmettent leurs substances conjointement à leur progéniture (la mère transmettant le sang et la chair, le père, les os, les cheveux, les ongles, etc.). L'autre, sans doute issue des réformes politiques imposées par la lignée des Tu'i Tonga pour maintenir leur pouvoir, postulant que la mère et elle seule «fabrique » toute la substance de son enfant. L'une et l'autre postulent que le sang/rang est transmis par la mère et en effet, à l'époque du contact, seule la mère transmettait son rang à son enfant (les chefs étant polygames, comme l'était aussi le Tu'i Tonga, seuls les enfants nés d'une mère de rang aristocratique étaient considérés comme aristocrates). 
La tradition orale rapporte un inceste entre l'un des tout premiers Tu'i Tonga, le proéminent Tu'itatu'i, et sa sœur, la Tu'i Tonga Fefine Latutama. Que cet inceste royal mythique soit une manière de souligner l'ampleur de la stature de Tu'itatu'i est peu douteux : cette transgression par excellence de l'ordre des humains, éminemment dangereuse pour le commun des mortels, prouve par elle-même la nature singulièrement divine du grand Tu'itatu'i. Cependant, cette union hiérogamique comporte probablement d'autres implications, comme le laisse penser le fait qu'il soit unique dans la généalogie royale et, d'autre part, que la Tu'i Tonga Fefine en cause soit, comme par hasard, la première de toutes les Tu'i Tonga Fefine. Tout se passe comme si l'union entre les deux souverains ne pouvait se faire, dans le mythe comme dans le rite, que sur le mode allusif, comme si la société fantasmait cette union, en évoquait l'idée mais sans lui donner une réalité plus sensible ou plus explicite.

Il paraît possible d'éclairer le sens de cette union sexuelle latente entre les deux souverains, le Tu'i Tonga et la Tu'i Tonga Fefine, en recourant à l'étude d'abord de ces configurations symboliques que sont les incestes premiers du mythe de création tongien puis à celle du grand rituel annuel de fertilité où la paire souveraine se trouvait impliquée.

Tout comme la figure d'Hikuleo, les incestes des grands commencements renvoient aux avatars de la bisexualité originaire du cosmos. L'inceste frère/sœur et tout particulièrement l'inceste gémellaire qui en est la forme dense constituent un paradoxe à partir duquel la pensée tongienne brode à l'envi. Dans le mythe, l'inceste frère/sœur fondateur est celui qui survient à la quatrième génération, entre Taufulifonua et Havealolofonua (géniteurs d'Hikuleo) : c'est, en effet, le premier inceste explicitement anthropomorphe, dont il est dit qu'il a lieu entre un homme et une femme, lesquels sont aussi des jumeaux de sexe opposé. Or, dans la mythologie tongienne, les jumeaux Taufulifonua et Havealolofonua sont présentés comme les initiateurs du premier acte sexuel (Gifford, $1924: 18)^{28}$. Autrement dit, l'inceste frère/sœur du temps des grands commencements renvoie à la division des sexes, certes, mais aussi à l'origine de la sexualité comme source et moteur de l'engendrement. $\mathrm{Au}$ temps des grands commencements, parait nous dire le mythe, dans un monde qu'on n'imagine que comme le produit d'engendrements, il a bien fallu passer par le un puis le deux pour arriver au multiple, arithmétique incontournable que l'on retrouve dans nombre de mythes de fondation polynésiens et qui rend l'inceste mythique des origines si productif. Mais l'inceste des grands commencements, générateur du monde et des grands dieux, est aussi porteur de mort et de destruction, comme paraît nous inviter à le penser le personnage crucial d'Hikuleo : au sein de la dynamique prolifique des engendrements, il est une sorte d'impasse reproductive, de cul-desac où le processus de filiation se gèle de luimême.

L'un des mythes les plus connus du monde polynésien, un mythe d'origine maori, raconte comment l'humanité naquit du geste de Tane (le premier homme) : en séparant ses parents, Papa (la terre) et Rangi (le ciel), étroitement enlacés dans une copulation sans fin, il mit fin à la nuit $(\mathrm{Po})$ en ouvrant la matrice de sa mère Papa où lui et ses frères étaient retenus prisonniers. Ainsi libérée de la fusion originaire, l'espèce humaine pourra croître et se multiplier (Reed, 1974: $5 s q)^{29}$. Comme Papa et Rangi, mais sous une autre forme, Hikuleo peut être considérée comme une figure involutive du processus filiatif, une sorte de retour au stade primordial intrautérin $^{30}$. Quant à l'inceste frère/sœur, à la fois condition de la (re)production de l'ordre sociocosmique et source de sa possible néantisation, d'un retour à la fusion matricielle originaire, il est conçu comme simultanément fondateur et destructeur de l'ordre social

$\mathrm{Au}$ temps du mythe, l'inceste frère/sœur est nécessaire à la création du monde et à la naissance des grands dieux mais, dans la vie sociale, il est interdit aux "vivants », aux humains. Or le chef suprême et sa sœur occupent un état intermédiaire entre le divin et l'humain, ou plutôt ils combinent en eux les deux états. Au temps du rite, durant la grande fête annuelle du 'inasi qui glorifie la fécondité générale, le Tu'i Tonga et la Tu'i Fefine constituent la version terrestre de la divinité mais une divinité clivée, sous la forme d'un frère et d'une sœur, à qui l'inceste est désormais interdit. Dans cette perspective, il n'est pas étonnant que durant le rite de l'intronisation royale qui célèbre conjointement l'avènement du chef suprême et le renouveau du cosmos, le bain

28. Dans Inalienable Possessions, The Paradox of Keeping-While-Giving, A. Weiner a montré que dans les sociétés polynésiennes où les relations frère/sœur font pourtant l'objet d'un puissant évitement, il existe tout un ensemble de productions sociales - récits, mythes, rites, généalogies, objets de valeur comme les nattes fines - qui font constamment allusion à ce qu'elle appelle la « sibling sexuality » (1992: 80).

29. Mais elle sera désormais mortelle.

30. À propos des « équivalences » mythiques entre cosmogenèse, anthropogenèse et ontogenèse, voir V. Valeri (1985:6). 
rituel de la Tu'i Tonga Fefine rejoue le paradoxe des grands commencements, l'idée de l'inceste sacré des origines. Simultanément, le bain rituel substitue au lien mère/enfant celui de tante paternelle/neveu. Tout dans la relation cruciale qui existe entre un enfant et sa tante paternelle montre qu'elle est un père au féminin (comme l'oncle maternel est une mère masculine). On peut alors voir aussi dans la seconde naissance du Tu'i Tonga, telle qu'elle est mise en scène dans le rite du bain royal, la césure imposée au groupe maternel par le groupe paternel et la captation, au nom de toute la société, de celui qui naît à la fonction de chef suprême.

Nous allons maintenant quitter la société tongienne du XIX ${ }^{\mathrm{e}}$ et nous rendre à l'autre extrémité du monde polynésien, aux îles Gambier, plus précisément à Mangareva où existe, sous diverses versions, un mythe d'origine qui traite très directement de l'accouchement.

\section{Comment les femmes apprirent à accoucher}

Voici comment la tradition orale de Mangareva raconte l'histoire de l'accouchement :

«Quand une femme devenait enceinte, on la reléguait dans une case particulière, située dans un lieu écarté. Elle devait y vivre, hors de tout contact avec les habitants, si ce n'est quelques femmes qu'on laissait auprès d'elle pour la servir. Les mois de gestation étaient comptés d'après les phases de la lune qui suivaient le commencement de la grossesse. Mais le processus naturel de l'accouchement était inconnu et à la neuvième lune, lorsque la femme commençait à souffrir des douleurs de l'enfantement, un vieux prêtre arrivait près d'elle, lui ouvrait le ventre et en retirait l'enfant tandis que la mère mourait. Cette opération se faisait avec un morceau de nacre fendu en biseau.

Elle ne cesse qu'à la suite du fait suivant. Un jour, une jeune femme de Mangareva se trouva enceinte, mais sans savoir elle-même au juste de quel homme. Elle alla faire part de sa situation à un sorcier, qui lui dit : "Ne te laisse pas ouvrir le ventre, je pars, et quand le moment de tes souffrances sera venu, tu me feras prévenir, et j'enverrai quelqu'un pour te soigner'. La jeune femme le lui promit.

Quelques mois se passèrent, et quand l'accouchement fut venu, un prêtre vint, comme d'habitude pour ouvrir le corps de la mère et y prendre l'enfant. Mais la jeune femme lui dit: "Attends un peu et reviens dans une heure". Le vieux prêtre y consentit; il s'en alla. Presque aussitôt après son départ, les douleurs de l'enfantement arrivèrent, et se rappelant alors ce que lui avait dit un de ses amants, la jeune femme s'écria : "Tagaroa ma rei ui vau". Immédiatement, deux autres femmes se présentèrent, portant chacune une gerbe d'herbe appelée aretu et l'une d'elle plaça la gerbe derrière le dos, tandis que l'autre étalait la sienne par terre. Il en résulta, dit la tradition, que les douleurs de la mère augmentèrent et qu'elle mit au monde son enfant tout naturellement.

C'est depuis lors que toutes les femmes adoptèrent cette façon d'accoucher et refusèrent de se laisser ouvrir le ventre par le sinistre prêtre. » (Caillot, 1914 : 149-150)

Bien entendu, l'expression «tout naturellement » est celle d'Eugène Caillot. Ce que nous dit le récit ci-dessus, c'est qu'il existait autrefois un état de société où les femmes ne savaient pas accoucher et où il leur fallait donc mourir, puisqu'on devait leur ouvrir le ventre pour permettre la naissance. Autrement dit, pour que l'enfant naisse, il fallait d'abord que la mère meure : en quelque sorte, l'accouchement n'existait pas. La tradition orale rapporte ici comment une femme apprend à enfanter, connaissance qui représente pour le mythe - dans ses diverses variantes - un progrès pour l'humanité.

Si j'ai choisi d'évoquer ce mythe mangarévien concernant l'invention de l'accouchement, c'est parce qu'il répond comme en écho aux pratiques entourant l'accouchement à Tonga : enfanter y est considéré, ici et là, comme un ensemble de connaissances secrètes qui appartiennent aux femmes et qu'elles se transmettent, pour le bien de tous.

\section{Interprétations}

Les mythes et les récits évoqués plus haut mettent en scène ce qu'on pourrait appeler, en reprenant les mots de Bernard Juillerat, des morceaux épars du « drame éclaté de l'œdipe » : non pas les figures, classiques dans nos sociétés, de l'inceste avec la mère ou du meurtre du père, mais celles de la fusion originaire, du retour au stade intra-utérin. C'est le coït éternel dans lequel se tiennent embrassés Rangi (le ciel) et Papa (la terre), empêchant leurs enfants de nâ̂tre, c'est Hikuleo, ce deux-en-un aussi clos sur lui-même qu'un œuf stérile. On pourrait y ajouter le coquillage bivalve dans lequel se tenait le dieu Ta'aroa avant que ce dernier ne l'ouvre pour créer le monde, selon le mythe d'origine tahitien. Images, diverses et variées, de la dualité originelle du cosmos, si caractéristiques des sociétés polynésiennes mais aussi représentations parallèles du stade primordial de l'univers socio-cosmique et de l'état de fusion originaire de l'espèce humaine.

Au-delà de ces configurations œdipiennes propres à ces sociétés, un premier constat s'impose. Comme l'avait remarqué Juillerat à propos des mythes yafar, ceux de Polynésie sont tournés bien davantage vers la "production de la 
génération », vers l'engendrement et l'enfantement que vers la sexualité, affichant une attention maniaque pour la mathématique reproductive des lignées :

« histoires créées par des adultes, les mythes montrent clairement qu'ils font grand cas de la reproduction alors que de la sexualité, ils s'amusent. » (Juillerat, $2001: 62-63$ )

Dans le mythe de création tongien, la sexualité n'est que brièvement évoquée à travers la paire des premiers jumeaux anthropomorphes, la grande œuvre du mythe est les travaux d'engendrement. On constatera ensuite que l'opposition nature/culture, telle du moins qu'elle s'est constituée en Occident, n'a pas cours ici. Si dualisme il y a, c'est, comme il a souvent été dit pour les sociétés de Polynésie, celui du jour, 'Aho, et de la nuit, $P o$, à condition de considérer que ces notions ne s'opposent justement pas comme le font le jour et la nuit dans nos sociétés ${ }^{31}$. L'univers socio-cosmique des Polynésiens est marqué de part en part par la dimension du sacré et l'opposition du jour et de la nuit y opère sur le registre de la métaphore. Si la "géographie du surnaturel » (Vernant, 1965: 116), telle qu'elle apparaît dans le mythe d'origine tongien, distingue bien des domaines comme le monde souterrain, la mer, les cieux, le Pulotu, tout y est soumis à l'ordre des dieux. Le monde du Po-nuit, c'est le monde enchanté des morts, peuplé d'une multitude d'esprits, d'ancêtres et de divinités. Mais le $P o$, c'est aussi l'origine des choses, c'est ce qui existait avant et qui existera après, c'est un monde antérieur et englobant, supérieur hiérarchiquement. Le monde du 'Aho-jour, c'est le monde visible, celui des « vivants » (mou'i), des hommes et de leurs actions, du temps présent. Mais c'est aussi un monde d'apparence, englobé par la nuit et pénétré par elle, puisque les esprits des morts, les ancêtres et les divinités peuvent y circuler sous des formes familières, visibles... ou invisibles. Le Po-nuit, c'est tout ce que les hommes ne voient pas, ne savent pas voir, ne comprennent pas : tout ce qui leur est inintelligible. C'est donc un monde éminemment dangereux pour les «vivants». Les cycles saisonniers comme celui des ignames, les mouvements des marées, ceux des astres, les règles et le corps féminin, la sexualité relèvent du Po-nuit. Ce n'est pas un monde sans loi où régnerait le chaos mais un univers régi par des lois qu'on ne connaît pas, qui ne sont pas du ressort des humains. Heureusement, entre le monde très sacré des origines, des morts et des dieux et celui des hommes, plus profane mais où le sacré s'insinue en se déguisant, des médiations sont possibles. Elles sont affaires de personnes comme les chefs - au premier rang desquels le Tu'i Tonga et la Tu'i Tonga Fefine - ou les prêtres, ou même, au sein de la parentèle, ceux qui représentent les ancêtres décédés, comme la tante paternelle.

On pourrait être tenté de lire l'opposition $P o$-nuit/'Aho-jour à la manière de la dichotomie nature/culture telle qu'elle est construite dans nos sociétés, en postulant l'équivalence du concept polynésien du $\mathrm{Po}$-nuit et de la catégorie occidentale de « nature », celle-ci étant comprise soit comme "le monde environnant» (sens le plus commun) soit comme un état infra social, au sens savant de Lévi-Strauss (puisque, dans le Po comme dans la nature, l'inceste est pratiqué). En somme, le surnaturel là-bas correspondrait au naturel dans nos sociétés. Cependant, cette manière de voir les choses souffre d'un inconvénient majeur. Dans la pensée de nos sociétés, la nature, qu'elle soit comprise comme « le monde environnant » et opposée à l'espèce humaine, ou entendue comme un "état infra-social» et opposée à la culture, est conçue, dans les deux cas, au sein d'une relation sujet/objet où elle occupe la place d'objet. Comme " monde environnant », la nature est destinée à être soumise au travail humain, comme " état infra-social", la nature se voit imposer des règles et des normes. Dans l'univers polynésien, l'opposition $\mathrm{Po}$ nuit/'Aho-jour, qui est à lire dans la perspective dumontienne de la hiérarchie et donc de la relation, nous invite à penser l'idée du passage, du va-et-vient, du mouvement qui va du plus au moins sacré. Ce ne sont pas seulement les notions du $P o$-nuit et du 'Aho-jour qui sont pertinentes dans l'imaginaire de ces sociétés, c'est aussi, c'est surtout, le passage de l'un à l'autre, du plus sacré au moins sacré... et retour. Or, à côté de tout ce qui - certains êtres, certains animaux, certains objets - est censé favoriser le passage entre le $P o$-nuit et le 'Aho-jour, il existe un élément-clé, une notion polymorphe, véritable interface entre les deux domaines, celle de fonиa (fenua), qui signifie à la fois « terre/pays », « tombe » et « placenta ».

31. Ces deux notions Po et 'Aho, en effet, ne constituent pas une opposition absolue dont les termes s'excluent l'un l'autre. Ils sont à lire comme Louis Dumont l'a montré pour le pur et l'impur en Inde, autrement dit au sein d'une hiérarchie, dans le sens qu'il donne à ce terme : l'une suppose l'autre, elles sont interdépendantes même si elles n'occupent pas, selon les perspectives, les mêmes niveaux de valeur. À l'âge classique ( $\mathrm{XVII}^{\mathrm{e}}-\mathrm{XIX}^{\mathrm{e}}$ siècles), la société polynésienne de Tonga peut être considérée comme relevant du type holiste et la distinction établie par Dumont entre statut (hiérarchique) et pouvoir (politique) y joue un rôle essentiel. 
C'est dans ce cadre conceptuel qu'il faut comprendre comment les Polynésiens du contact concevaient les procès associés à l'enfantement et à la naissance. Le cycle de vie, qui n'a ni début ni fin, comporte deux passages obligés entre le monde du Po-nuit - le lieu-origine de tout - et le monde du' Aho-jour, là où vivent les humains : le placenta et la tombe, « pays » respectifs du foetus et du décédé. On s'en souvient ( $c f$. supra), nombre de mythes polynésiens font du corps féminin et en particulier de la matrice féminine, du placenta ou du vagin, des moyens de médiation entre le monde très sacré des esprits/ancêtres/ divinités et celui des humains. Encore aujourd'hui, les cheveux du nouveau-né sont appelés d'un terme qui signifie « les cheveux de l'esprit », le tout petit enfant étant censé issu du monde surnaturel. Quant au récit de l'apprentissage de l'accouchement, dans la tradition mangarévienne, que raconte-il d'autre que ce que disent de l'enfantement les pratiques tongiennes: accoucher est un savoir, un ensemble de connaissances secrètes qui appartiennent aux femmes et qu'elles se transmettent pour le plus grand bien de la société humaine. En tongien, comme dans la plupart des langues polynésiennes, « engendrer », « accoucher » et « naître » se dit d'un seul et même terme, celui de fanau. Événement unique raconté par les mythes et les rites, l'engendrement, l'enfantement et la naissance sont ici considérés comme un ensemble de pratiques, de savoirs et de savoir-faire qui met à la portée des hommes ce qui vient du monde des dieux : un travail qui est l'affaire de tous, hommes et femmes, chefs et ancêtres, bref un événement cosmique qui « fait société ».

Dans les représentations de l'engendrement et de la filiation se jouent ce que Bernard Juillerat appelle les questionnements ontologiques de l'homme vivant en société. Là, se cristallise une part non négligeable des craintes, des angoisses, des fantasmes, associés à la lente élaboration de la vie en société. Pour leur part, les productions culturelles de Polynésie témoignent de ce que les procès associés à l'enfantement - et le lien mèreenfant qui en découle - constituent des objets d'appropriation sociale puissamment investis par l'imaginaire des sociétés concernées.

\section{BIBLIOGRAPHIE}

Bloch Maurice et Jean H. BLoch, 1980. Women and the Dialectics of Nature in Eighteenth-century French Thought, in MacCormack Carole and Marilyn Strathern (eds), Nature, Culture and Gen- der, Cambridge, Cambridge University Press, pp. 25-41.

BotT Elizabeth, 1982. Tongan Society at the Time of Captain Cook's Visits, Wellington, The Polynesian Sociéty, Memoir 44.

CaIllot Eugène, 1914. Mythes, légendes et traditions des Polynésiens, Paris, Ernest Leroux.

FAssin Éric, 2008. L'empire du genre. L'histoire politique ambiguë d'un outil conceptuel, L'Homme 187-188, 3-4, pp. 375-392.

Descola Philippe, 1986. La Nature domestique : symbolisme et praxis dans l'écologie des Achuar, Paris, Éditions de la maison des sciences de l'homme.

—, 2005. Par-delà nature et culture, Paris, Gallimard.

Dixon R.B., 1964. The Mythology of all Races, Oceanic, New York, Cooper Square Eds.

DouAIRE-MArsaudon Françoise, 1998. Les Premiers fruits. Parenté, identité sexuelle et pouvoirs en Polynésie occidentale (Tonga, Wallis et Futuna), Paris, CNRS-Éditions, Éditions de la maison des sciences de l'homme.

_, 2002. D'un sexe, l'autre. Le rituel du kava ou la reproduction de l'identité masculine en Polynésie, L'Homme 157, pp. 7-34.

—, 2004. Book review forum. Bernard Juillerat's book, Penser l'imaginaire. Essais d'anthropologie psychanalytique, Journal of Ritual Studies 18 (1), pp. 129-166.

Dumont Louis, 1966. Homo hierarchicus. Le système des castes et ses implications, Paris, Gallimard.

Dumont d'Urville Jules, 1833. Voyage de la corvette l'Astrolabe, exécuté par ordre du roi... Histoire $d u$ voyage, 5 vol. et 2 vol. d'atlas, Paris, Tastu.

Foucault Michel, 1976. Histoire de la sexualité. La volonté de savoir, vol. 1, Paris, Gallimard.

GIFFORD Edward, 1924. Tongan Myths and Tales, Honolulu, Bernice P. Bishop Museum, Bulletin 8.

—, 1929. Tongan Society, Honolulu, Bernice P. Bishop Museum, Bulletin 61.

GODELIER Maurice, 1982. La production des grands hommes, Paris, Fayard.

Hanson F. Allan, 1982. Female Pollution in Polynesia, Journal of the Polynesian Society 91, pp. 335-381.

HÉRITIER Françoise, 1981. L'exercice de la parenté, Paris, Gallimard-Le Seuil.

Juillerat Bernard, 2001. Penser l'imaginaire. Essais d'anthropologie psychanalytique, Lausanne, éditions Payot.

Kamakau Samuel M., 1991. Ka Po'e Kahiko. The People of Old, Honolulu, Bishop Museum Press.

LAQUEUR Thomas, 1992. La fabrique du sexe. Essai sur le corps et le genre en Occident, Paris, Gallimard.

LÉvi-STRAuss Claude, 1967. Les structures élémentaires de la parenté, La Haye, Mouton \& maison des sciences de l'homme. 
—, 1983. L'identité. Séminaire dirigé par Claude LéviStrauss, Paris, Presses universitaires de France.

MacCormack Carole and Marilyn Strathern, 1980. Nature, Culture and Gender, Cambridge, Cambridge University Press.

Mathieu Nicole-Claude, 1991. Homme-culture et femme-nature ? in L'anatomie politique. Catégorisations et idéologies de sexe, Paris, Côté-Femmes, pp. 43-61.

MARTIN John, 1981 [1817]. Tonga Islands. William Mariner's Account. An Account of the Natives of the Tonga Islands, in the South Pacific Ocean. With an original Grammar and Vocabulary of their Language. Compiled and Arranged from the Extensive Communications of $\mathrm{Mr}$ William Mariner, several years Resident in Those Islands, Tonga, Vava'u Press.

ORTNER Sherry, 1974. Is Female to Male as Nature is to Culture, in Rosaldo Michelle Z. and Louise Lamphere (eds), Woman, Culture and Society, Standford, Stanford University Press.

ReED A. W., 1974. Myths and Legends of Polynesia, Wellington, Sydney, London, A.H. \& A.W. Reed.

REITER François, 1907. Traditions tonguiennes, Anthropos 2, pp. 230-240.
SAHLINs Marshall, 1985. Islands of History, Chicago, London, UCP.

SAura Bruno, 2008. Tahiti Mā'ohi. Culture, identité, religion et nationalisme en Polynésie française, Papeete, éditions-Tahiti Au Vent des îles.

Strathern Marilyn, 1980. No Nature, No Culture: the Hagen Case, in MacCormack Carole and Marilyn Strathern (eds), Nature, Culture and Gender, Cambridge, Cambridge University Press, pp. 174-222.

TAHON Marie-Blanche, 1995. Le don de la mère, Anthropologie et Société 19, 1-2, pp. 139-155.

VALERI Valerio, 1985. Kingship and Sacrifice. Ritual and Society in Ancient Hawaii, Chicago and London, University of Chicago Press.

Vernant Jean-Pierre, 1965. Mythe et pensée chez les Grecs. Études de psychologie historique, nouvelle édition, revue et augmentée, Paris, La Découverte.

WeIner Annette, 1992. Inalienable Possessions, The Paradox of Keeping-While-Giving, Berkeley, Los Angeles, Oxford, University of California Press.

Williamson Robert W., 1933. Religious and Cosmic Beliefs of Central Polynesia, 2 vol., Cambridge, Cambridge University Press. 
